\title{
Accuracy Improvement in the Nano-displacement Measurement Based on the Doppler-Interferometry Method by Cross-talk Reduction
}

\author{
Saeed Olyaee Shahram Mohammad Nejad \\ Optoelectronic and Laser Laboratory, Department of Electrical Engineering, \\ Iran University of Science and Technology (IUST) \\ s_olyaee@srttu.edu \\ shahramm@iust.ac.ir
}

\begin{abstract}
In this paper, accuracy improvement in the displacement measurement systems using Dopplerinterferometry method is presented. The cross-talk error reduction methods are also discussed. Based on the mentioned method, a new nano-displacement measurement system is designed. In the designed system, the signal to leakage ratio for moving targets in the range of $-20 \mathrm{~m} / \mathrm{s}$ to $+20 \mathrm{~m} / \mathrm{s}$ is increased to $55 \mathrm{~dB}$. The measured phase detection error is equal to 7.9 degree in the normal condition, and after the cross-talk error reduction it is reduced to 0.11 degree. As a result, the displacement measurement error is decreased from $6.9 \mathrm{~nm}$ to $0.1 \mathrm{~nm}$.
\end{abstract}

Keywords: cross-talk error, Doppler-interferometry, nano-displacement, He-Ne laser.

\section{1- Introduction}

In optical instruments such as laser interferometer, various parameters affect the accuracy. The error sources in the laser interferometer systems include the non-linearity in the optical section, intensity fluctuation and laser frequency instability, environment turbulences, misalignment in the optical instruments, systematic error in the phase detection, and cross-talk error. Several error sources are considered as linear errors that can be simply reduced or eliminated [1-3]. But the cross-talk error especially in the heterodyne method produces the non-linearity error. This effect on the laser range finders based on the phase shift method is reported in [4].

Heterodyne methods such as Dopplerinterferometry compared to homodyne methods provide more signal to noise ratio and easy alignment. Because of using the two separated beams in the heterodyne method, the non-linearity errors especially cross-talk error is dominance [5-7]. By combining the reference and the measurement fields, the phase error is obtained from

$\Phi_{e r r}=\theta_{o}-\Phi_{o}=\tan ^{-1}\left(-I_{o l} / I_{o t}\right)$

where $\theta_{o}$ is the measurement phase, $\Phi_{o}$ is the real phase, $I_{o t}$ and $I_{o l}$ are the main signal and leakage amplitudes, respectively. The diagram of the main signal $I_{o t}$, leakage $I_{o l}$, and the measurement signal $I_{\text {om }}$ in the heterodyne system is shown in figure 1. The amplitude of the measurement signal is given by

$I_{o m}=\sqrt{I_{o l}^{2}+I_{o t}^{2}+2 I_{o l} I_{o t} \cos \Phi_{e r r}}$

According to Eq.2 and figure 1, the phase error and hence the systematic errors will increase by increasing the leakage amplitude.

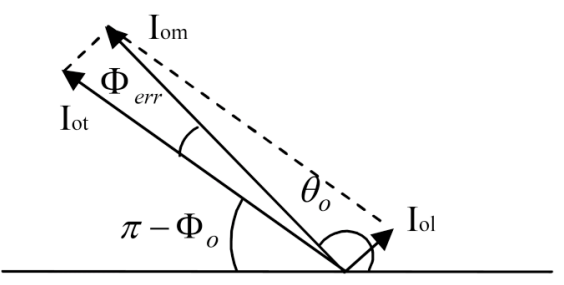

Fig.1: Diagram of the main signal, leakage, and measurement signal in the heterodyne system.

\section{2- Cross-talk error in the Doppler- interferometer}

Two main sources produce the cross-talk error in the laser Doppler-interferometer. The first one includes the electrical induction between the reference and measurement paths. The other one is due to the unwanted electromagnetic leakage of two modes. The output beam contains two orthogonal linearly components with small difference between their frequencies. As a result, the laser output can be used as a reference beam and measurement beam, separately. Ideally a polarizing beam splitter (PBS) can separate the laser output into two beams with different wavelengths and polarizations. But, in practical systems, there exist a non-orthogonality between the linear beam polarization and elliptical polarization of the individual beams. And hence, after separation, a small fraction of each mode is available in the other.

Figure 2 shows the block diagram of the velocity and nano-displacement measurement system based on the laser Doppler-interferometry. The current to voltage converter (IVC), pre-amplifier, and comparator circuits are similar in the $K_{1}$ and $K_{2}$ boxes. 


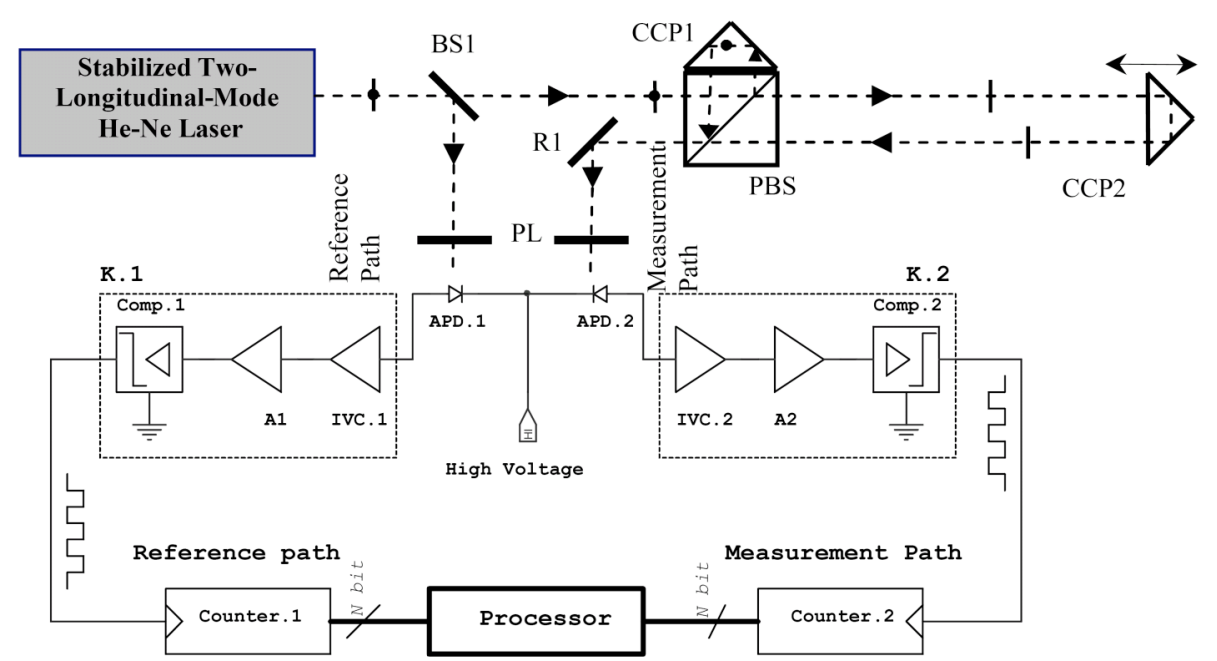

Fig. 2: Block diagram of the velocity and nano-displacement measurement system. BS: beam splitter, PBS: polarizing beam splitter, CCP1: corner cube prism1 (reference) and CCP2: corner cube prism2 (target).

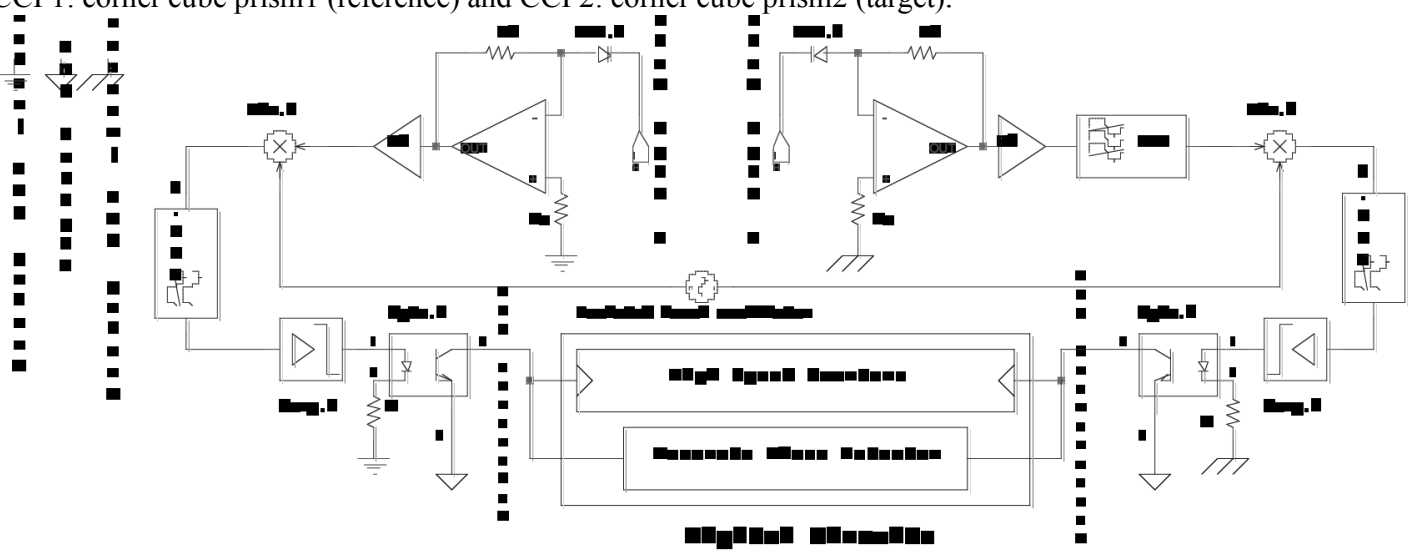

Fig. 3: Schematic diagram of the electronic section of the velocity and nano-displacement measurement system with noise and cross-talk error reduction.

The photocurrent of the APD at the measurement path $\left(A P D_{2}\right)$ is given by

$I_{A P D_{2}}=k\left[E_{1} \cos \left(2 \pi v_{1} t\right)+E_{2} \cos \left(2 \pi v_{2}^{\prime} t+\varphi+\Phi\right)\right]^{2}$

where $v_{1}$ and $v_{2}$ are the frequencies of two modes $\left(v_{o}=v_{2}-v_{1}\right), \phi$ is the initial phase between the two, $\Phi$ is the phase shift due to difference in the optical paths, and $v_{2}^{\prime}{ }_{2}$ is the reflected beam frequency of the target mirror $\left(\mathrm{CCP}_{2}\right)$. The reflected beam frequency is obtained as

$v_{2}^{\prime}=v_{2} \pm 2 n \bar{V} / \lambda_{2}$

where $\bar{V}$ is the velocity of the target and $n$ is the refractive index of the medium along the laser path.

Considering the cross-talk error, a fraction of reference mode (with $v_{1}$ frequency) includes in the measurement mode. The leakage frequency, therefore, is shifted by an amount $\pm 2 n \bar{V} / \lambda_{1}$, $\left(v^{\prime}{ }_{1}=v_{1} \pm 2 n \bar{V} / \lambda_{1}\right)$. As mentioned above, the leakage amplitude is much smaller than the main signal amplitude $\left(\widetilde{E}_{1} \ll E_{1}\right)$. Hence, the photocurrent of avalanche photodiode (Eq.3) is corrected as

$I_{A P D_{2}}^{\prime}=k\left[\begin{array}{c}E_{1} \cos \left(2 \pi v_{1} t\right)+\widetilde{E}_{1} \cos \left(2 \pi v_{1}^{\prime} t+\Phi\right) \\ +E_{2} \cos \left(2 \pi v_{2}^{\prime} t+\varphi+\Phi\right)\end{array}\right]^{2}$

In addition, the high frequencies are eliminated by $A P D_{2}$. As a result, the Eqs. 3 and 5 are simplified to

$I_{A P D_{2}}=2 k E_{1} E_{2} \cos \left(2 \pi\left(\gamma_{o} \pm 2 n \bar{V} / \lambda_{2}\right)+\varphi+\Phi(t)\right)(6)$

$I^{\prime}{ }_{A P D_{2}}=2 k E_{1} \widetilde{E}_{1} \cos \left(2 \pi\left(+2 n \bar{V} / \lambda_{1}\right)+\Phi(t)\right)+$

$$
\begin{aligned}
& +2 k E_{1} E_{2} \cos \left(2 \pi\left(v_{o} \pm 2 n \bar{V} / \lambda_{2}\right)+\varphi+\Phi(t)\right)+ \\
& +2 k \widetilde{E}_{1} E_{2} \cos \left(2 \pi\left(v_{o} \pm 2 n \bar{V}\left(1 / \lambda_{2}-1 / \lambda_{1}\right)\right)+\varphi\right)
\end{aligned}
$$

In Eq.7, the first and third terms are the crosstalk components and the second term (similarly to Eq.6) is the main signal. The last term can be rewritten as $\quad 2 k \widetilde{E}_{1} E_{2} \cos \left(2 \pi v_{o}(1 \pm 2 n \bar{V} / C)+\varphi\right), \quad$ where $\left|v_{2}-v_{1}\right| / C$ is equal to inverse of the synthetic wavelength, $\lambda_{s}$. 


\section{3- The cross-talk error reduction method}

As is shown in figure 2, a stabilized two-longitudinalmode He-Ne laser having $632.8 \mathrm{~nm}$ wavelength and $152 \mathrm{MHz}$ free spectral range is used. Therefore, the maximum measurable velocity is equal to $\bar{V}_{\max }=\lambda v_{o} / 2 n=48.1 \mathrm{~m} / \mathrm{s}$. By approaching the target $\left(\mathrm{CCP}_{2}\right)$ close to the system, for example with $1 \mathrm{~m} / \mathrm{s}$ velocity, the frequencies of Eq.7 are obtained as $3.16 \mathrm{MHz}, 155.16 \mathrm{MHz}$, and $152 \mathrm{MHz}$, respectively.

The schematic diagram of the electronic section of the velocity and nano-displacement measurement system with noise and cross-talk error reduction techniques is shown in figure 3 . The analog ground (including reference and measurement grounds) and digital ground are isolated. Also, two isolated high voltage power supplies are used for APD biasing.

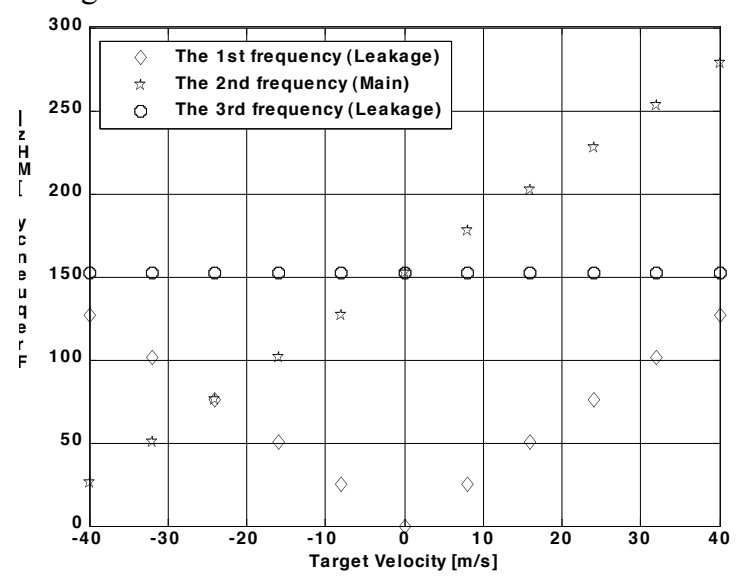

Fig.4: The measurement frequencies (main and leakage) in terms of the target velocity.

The measurement frequencies in terms of the target velocity is shown in figure 4. By using a band pass filter (BPF) having cut-off frequencies equal to $75 \mathrm{MHz}$ and $230 \mathrm{MHz}$, the leakage signal is considerably reduced. Although, the maximum measurable velocity is limited to $\pm 20 \mathrm{~m} / \mathrm{s}$, but the cross talk error is reduced dramatically. The input and output signals of the measurement band pass filter (BPF) is shown in figure 5. The output signal of the measurement BPF is mixed with the local oscillator signal $\left(f_{l}=233 \mathrm{MHz}\right)$. The new frequencies in terms of the target velocity is shown in figure 6 (the bold line is the main signal frequency, the dashed lines are the leakage frequencies, and the dotted line is the image frequency). The amplitude of the measurement signal for velocities $\pm 20 \mathrm{~m} / \mathrm{s}$ and $\pm 5 \mathrm{~m} / \mathrm{s}$ is shown in figure 7. The leakage and image frequencies are finally eliminated by a low pass filter whose cut-off frequency is $150 \mathrm{MHz}$. The output signals of the LPFs passed through opto-coupler isolators and exerted to digital section. In this section, an accurate phase detector measured the initial and final phases between the reference and measurement signals. Also, a high speed counter measured the shifted frequency due to the Doppler effect. The output of counter 1 is proportional to the free spectral range of the laser (the frequency difference between the two modes). But the output of counter 2 changes proportional to the speed of the target. Comparing the counter's outputs, the direction is detected.
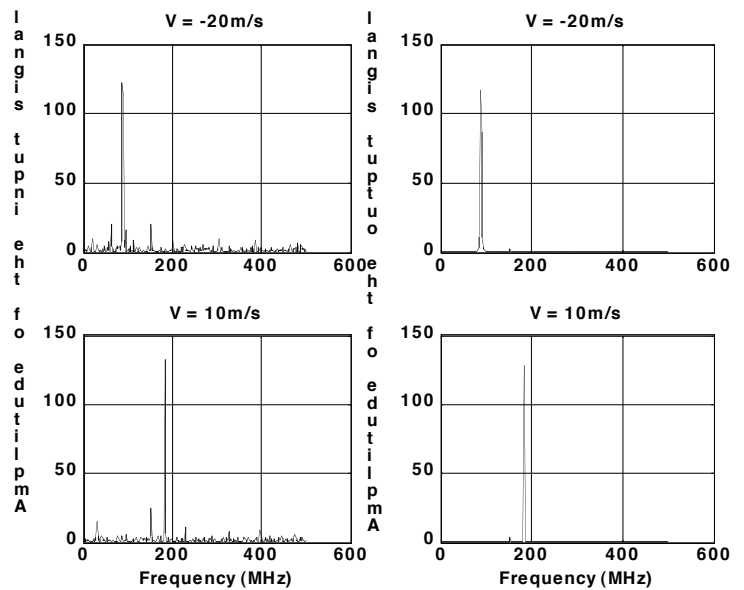

Fig.5: Left- the input and right- the output signals of the measurement band pass filter (BPF).

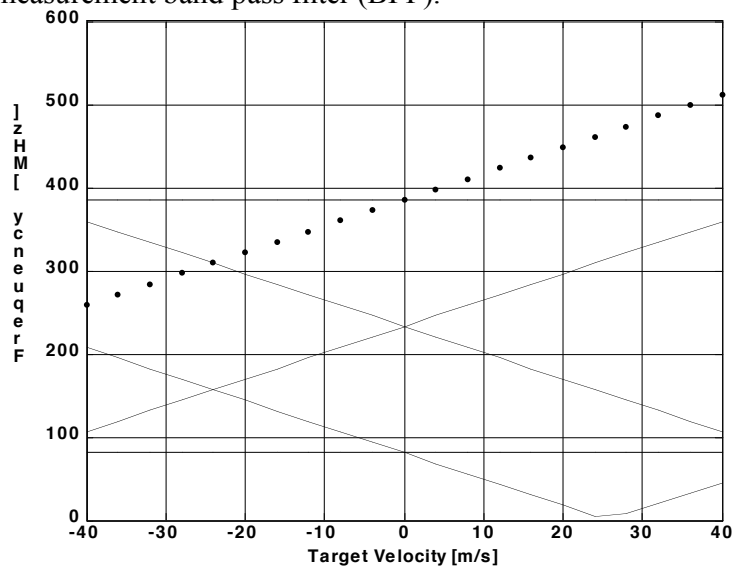

Fig.6: The output frequencies of the measurement mixer (Mix.2) in terms of the target velocity. The bold line is the main signal frequency, the dashed lines are the leakage frequencies, and the dotted line is the image frequency.

\section{4- Results}

In this system the cross-talk error is reduced by, i. Coupling reduction between reference and measurement paths by using a proper shielding, grounding and using the two isolated power supplies.

ii. Noise reduction by using an electromagnetic interference shield.

iii. Laser power reduction. 
iv. Using the band pass and low pass filters.
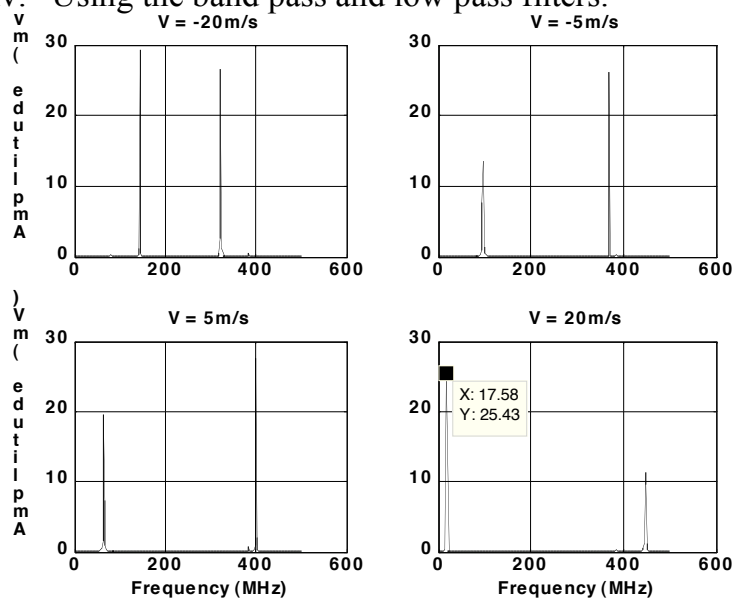

Fig.7: The amplitude of the measurement signals after the noise and cross-talk reduction $( \pm 20 \mathrm{~m} / \mathrm{s}$ and $\pm 5 \mathrm{~m} / \mathrm{s}$ ).

The measurement signal amplitude and phase detection error in terms of leakage amplitude is depicted in figure 8 . In the normal condition, the amplitude of the main signal and total leakage are $398.7 \mathrm{mV}$ and $55.3 \mathrm{mV}$, respectively. As a result, according to Eq.8, the signal to leakage ratio (SLR) is increased considerably, and is given by

$S L R=I_{o t} /\left(\sum_{i=1}^{n} I^{2}{ }_{o l_{i}}\right)^{1 / 2}$

where $I_{o t}$ is the main signal amplitude and $I_{o l_{i}}$ is the amplitude of the $i^{\text {th }}$ leakage. Based on the mentioned methods, the signal to leakage ratio is reached to $55 \mathrm{~dB}$. The displacement resolution is obtained as

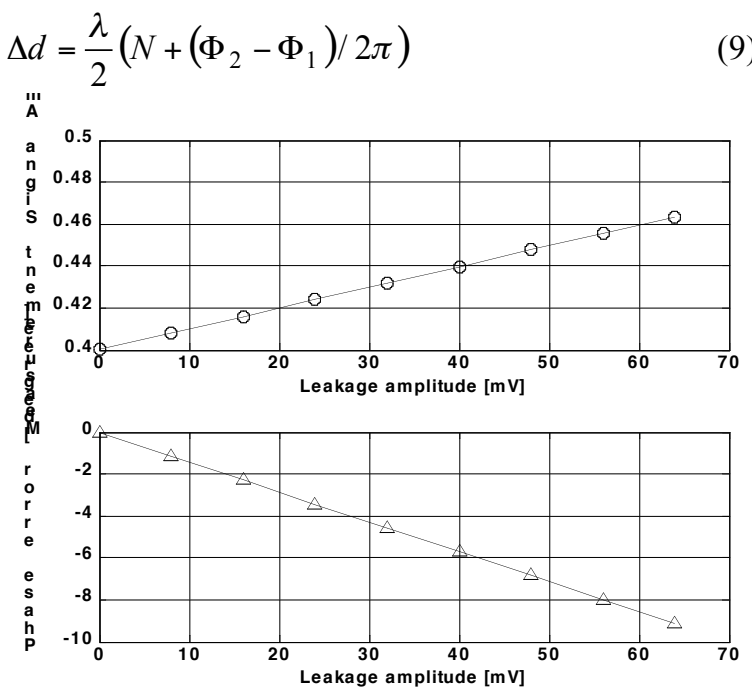

Fig.8: The measurement signal amplitude and phase detection error in terms of leakage amplitude.

where $\lambda$ is the central wavelength, $N$ is the shifted frequency due to the Doppler effect, $\Phi_{1}$ and $\Phi_{2}$ are the initial and final phases between the reference and measurement signals, respectively. Since the detected phase is changed by leakage, so the error in the nanometric displacement measurement $\left(\delta_{d}\right)$ is expressed as

$\delta_{d}=\frac{\lambda}{2}\left[\tan ^{-1}\left(I_{o l} / I_{o t}\right) / 2 \pi\right]$

The phase detection error before and after the noise and cross-talk reduction are 7.9 and 0.11 degree, respectively. The displacement error is limited to $0.1 \mathrm{~nm}$; despite the fact that it was $6.9 \mathrm{~nm}$ before the cross-talk error reduction. The summary of the designed system's performances is described in table.1.

Table.1: The summary of the typical performance.

\begin{tabular}{lll}
\hline Parameter & $\begin{array}{l}\text { Before the } \\
\text { noise and } \\
\text { cross-talk } \\
\text { reduction }\end{array}$ & $\begin{array}{l}\text { After the } \\
\text { noise and } \\
\text { cross-talk } \\
\text { reduction }\end{array}$ \\
\hline Main signal amplitude & $398.7 \mathrm{mV}$ & $441.3 \mathrm{mV}$ \\
Total leakage amplitude & $55.3 \mathrm{mV}$ & $0.81 \mathrm{mV}$ \\
SLR & $17 \mathrm{~dB}$ & $55 \mathrm{~dB}$ \\
Phase detection error & $7.9^{\circ}$ & $0.11^{\circ}$ \\
Displacement error & $6.9 \mathrm{~nm}$ & $0.1 \mathrm{~nm}$ \\
\hline
\end{tabular}

\section{References}

[1] J.F. Meyers, J. W. Lee and R. J. Schwartz, "Characterization of measurement error sources in Doppler global velocimetry”, Meas. Sci. Technol. 12, 357-368, 2001.

[2] Sutton C M, "Nonlinearity in the length measurement using heterodyne laser Michelson interferometry", J. Phys. E: Sci. Instrum. 20 1290-2, 1987.

[3] Hou W and Wilkening G, "Investigation and compensation of the nonlinearity of heterodyne interferometers", Prec. Eng. 14 91-8, 1992.

[4] S.Mohammad Nejad and S.Olyaee , "Cross-Talk and Intermediate Frequency Deviation Effects on Phase-Shift Range Finder", Iranian Journal of Science and Technology, Vol.26, No.B4, PP. 647-654, 2002.

[5] Patterson S and Beckwith J, "Reduction of systematic errors in heterodyne interferometric displacement measurement", Proc. 8th IPES Conf. (Compiegne, France) pp 101-4, 1995.

[6] Rosenbluth A E and Bobroff N, "Optical sources of nonlinearity in heterodyne interferometers", Precision Eng. 12 7-11, 1990.

[7] T.Eom, T.Y.Choi, K.Lee, H.Choi and S.Lee, "A simple method for the compensation of the nonlinearity in the heterodyne interferometer", Meas. Sci. Technol. 13, 222-225, 2002. 